\title{
Direct observation of high velocity current induced domain wall motion
}

\author{
L. Heyne, ${ }^{1}$ J. Rhensius, ${ }^{1, a)}$ A. Bisig, ${ }^{1}$ S. Krzyk, ${ }^{1}$ P. Punke, ${ }^{1}$ M. Kläui, ${ }^{1, b)}$ L. J. Heyderman, ${ }^{2}$ \\ L. Le Guyader, ${ }^{3}$ and F. Nolting ${ }^{3}$ \\ ${ }^{1}$ Fachbereich Physik, Universität Konstanz, Universitätsstr. 10, D-78457 Konstanz, Germany \\ ${ }^{2}$ Laboratory for Micro- and Nanotechnology, Paul Scherrer Institut, CH-5232 Villigen PSI, Switzerland \\ ${ }^{3}$ Swiss Light Source, Paul Scherrer Institut, CH-5232 Villigen PSI, Switzerland
}

(Received 19 November 2009; accepted 18 December 2009; published online 22 January 2010)

\begin{abstract}
We study fast vortex wall propagation in Permalloy wires induced by $3 \mathrm{~ns}$ short current pulses with sub 100 ps rise time using high resolution magnetic imaging at zero field. We find a constant domain wall displacement after each current pulse as well as current induced domain wall structure changes, even at these very short timescales. The domain wall velocities are found to be above $100 \mathrm{~m} / \mathrm{s}$ and independent of the domain wall spin structure. Comparison to experiments with longer pulses points to the pulse shape as the origin of the high velocities. (c) 2010 American Institute of Physics. [doi:10.1063/1.3291067]
\end{abstract}

Current-induced domain wall (DW) motion has become a very active field of research due to the exciting physics involved in the interaction between current and magnetization, ${ }^{1-4}$ but it could also pave the way to innovative applications. ${ }^{5}$ While qualitatively the motion can be attributed to the transfer of angular momentum from the conduction electrons to the magnetization, the details are unclear and large discrepancies in the experimentally observed velocities as well as between experimental results and theoretical predictions exist.

The spin polarized current interacts with the magnetization via the so-called adiabatic and nonadiabatic spin torque. ${ }^{3,4}$ This can analytically be described by the micromagnetic Landau-Lifschitz-Gilbert equation of motion extended by two spin torque terms. ${ }^{3,4}$ The current density enters this equation in the form of the parameter $u$ :

$$
u=\frac{P \mu_{B}}{e M_{s}} j,
$$

that has dimensions of a velocity. For Permalloy $u[\mathrm{~m} / \mathrm{s}] \approx 29 \cdot j\left[10^{12} \mathrm{~A} / \mathrm{m}^{2}\right]$ assuming a saturation magnetization of $M_{s}=8 \times 10^{5} \mathrm{~A} / \mathrm{m}$ and a spin polarization of the conduction electrons of $P \approx 0.4{ }^{6}$

For large scale displacements the average DW velocity $\langle v\rangle$ below the Walker breakdown is assumed to directly scale with $u:\langle v\rangle=\frac{\beta}{\alpha} u .{ }^{4}$ Here $\alpha$ denotes the damping constant and $\beta$ the nonadiabaticity parameter. Above the breakdown, $\langle v\rangle$ is expected to approach $u$. The initial velocity $v$ of the DW when current is injected is $v=\frac{1+\alpha \beta}{1+\alpha^{2}} u \approx u .^{7}$ Measuring the DW velocity as a function of time could thus be used to gain information about the ratio $\beta / \alpha$. Whether the above relations are applicable for real experimental results is discussed controversially, since effects relevant in the experiment such as extrinsic pinning are not considered in the model.

The DW velocities observed in previous experiments seem to be split in two regimes. Either velocities comparable to the spin transfer momentum rate $u \approx 100 \mathrm{~m} / \mathrm{s}$ are found ${ }^{8,9}$ or velocities of a few meter per second, hence much lower than $u .^{10-13}$ The origin of this discrepancy is to the present

\footnotetext{
a) Also at LMN, Paul Scherrer Institut, CH-5232 Villigen, PSI.

${ }^{b)}$ Electronic address: mathias.klaeui@uni-konstanz.de.
}

date not clear. Differences in the magnetic properties and the roughness of either the edges or the surfaces of the nanowires resulting from the deposition process and the seed layers have been suggested. ${ }^{8}$ This could result in different extrinsic pinning strength and thus affect the DW velocity.

In this letter, we present high velocity DW motion induced by short current pulses with ultrashort rise times at zero field. Using a fast photodiode and a short laser pulse, we inject current pulses with approximately 3 ns length and a steep rise time of less than $100 \mathrm{ps}$. We show that the pulses not only displace the DW but also induce DW transformations. Comparing the results to DW displacements induced by microsecond pulses, we find that fast DW velocities are only observed for short pulses with fast rise times.

Permalloy $\left(\mathrm{Ni}_{80} \mathrm{Fe}_{20}\right)$ wires with a $2 \mathrm{~nm}$ capping layer of gold that are $1000 \mathrm{~nm}$-wide and $40 \mathrm{~nm}$-thick were fabricated on naturally oxidized high-resistive Si substrates by electronbeam lithography and a two step lift-off process. ${ }^{14}$ The wires are contacted by Au pads on both ends for current injection. An image of the wire with the contacts is shown in Fig. 1(a).

X-ray photoemission electron microscopy (XPEEM) employing X-ray magnetic circular dichroism is used to image the magnetization. ${ }^{15}$ The sample is at a potential of $15 \mathrm{kV}$ compared to ground and connected via cables not suitable for HF injections which prohibits the use of a normal pulse injection setup. Therefore, we employ a combination of femtosecond laser pulses with a photodiode on the sample holder to generate pulses with a rise time below those achievable using pulse generators.

The wire is connected to a fast photodiode that is biased by a dc voltage $U_{\mathrm{b}}$. A $100 \mathrm{fs}$ laser pulse focused on the diode creates free charge carriers that are then injected into the structure. The pulse of the laser is broadened by the diode so that the current pulse through the wire is longer. To determine the pulse shape, a special technique is used: Instead of a single laser pulse, a continuous pulse train is injected and by synchronizing the laser pulses to the synchrotron x-rays, a pump-probe setup is designed. ${ }^{16}$ With this setup we can study the temporal response of the sample to the laser-induced current pulse by changing the delay between the laser pump pulse and the $\mathrm{x}$-ray probe pulse. The current inside the Permalloy wire creates a potential gradient along the current path. Since the left part of the structure 

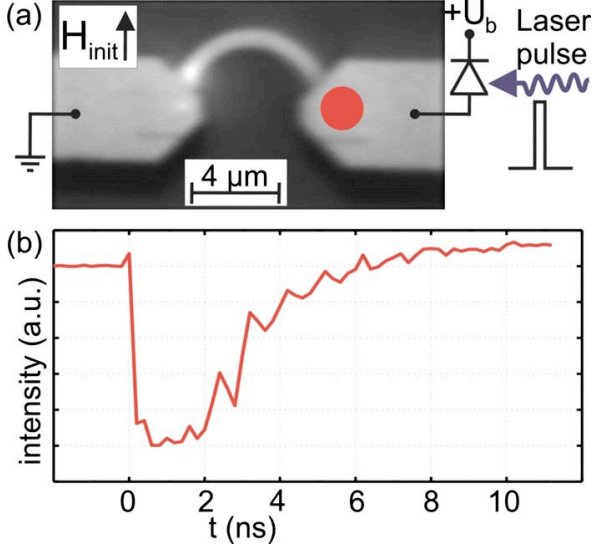

FIG. 1. (Color online) (a) Image of the structure and schematic setup of the experiment. The direction of the field $H_{\text {init }}$ used to nucleate a DW is shown. The sample (1000 nm wide and $40 \mathrm{~nm}$ thick Permalloy curved wire) is contacted to a diode. A laser pulse is focused on the diode and the created charge carriers are than injected into the wire. The red circle marks the region where the intensity scan in (b) was measured. (b) Intensity of the marked region in (a) vs time delay. During the current pulse the sample potential changes resulting in a decrease in the intensity and this allows us to deduce the pulse shape.

[Fig. 1(a)] is fixed to the sample ground, the potential during the current pulse will increase with increasing distance from the ground pad. The photoemission electron microscope detects secondary electrons of a selected energy range and the current-induced potential shift results in a change of the detected electron intensity. Figure 1(b) shows the intensity as a function of the time delay between pump and probe pulse for the right hand side pad, which is connected to the diode [see red circle in Fig. 1(a)]. The pulse has an extremely fast rise time, less than our time resolution of $100 \mathrm{ps}$, which would be hard to generate using a purely electronic pulse generator setup. The pulse fall time is approximately $1 \mathrm{~ns}$ and the total pulse length is of about $3 \mathrm{~ns}$. The repetition rate of the laser is $5 \mathrm{MHz}$, corresponding to a $200 \mathrm{~ns}$ period. In total $4 \mathrm{~mA}$ are drawn from the power supply $U_{\mathrm{b}}$, when the laser is on. This corresponds to a peak current density during the pulse of about $j=4 \times 10^{12} \mathrm{~A} / \mathrm{m}^{2}$. Due to nonlinearities in the delay scan shown in Fig. 1(b), the error for the current density is significant and estimated to be about $50 \%$.

To create a DW prior to the current injections, an external magnetic field is applied in vertical direction $\left(H_{\text {init }}\right.$ in Fig. 1). After reducing the field to zero, a DW is formed at the center of the wire. An XPEEM image of the initial configuration is presented in Fig. 2(a). The top image shows a vortex wall (VW) at the wire center. To visualize the spin structure, the inset in the lower left shows a micromagnetic simulation of a VW confined in a wire with the same configuration. The magnetization curls around the center of the wall, the so-called vortex core, where the magnetization points out-of-plane to reduce the exchange energy. ${ }^{17}$ Once the initial configuration is imaged, single current pulses are injected into the structure. The result of a series of injections is presented in Fig. 2. Current pulses were injected between adjacent images (from top to bottom). After five injections, the DW is displaced by about $2 \mu \mathrm{m}$. In order to visualize each displacement, contrast profiles across the DW indicated by the yellow line in the bottom image of Fig. 2(a) are presented in Fig. 2(b). After the third current pulse, the left end of the wall has not moved and it seems to be pinned, whereas the right side of the DW continues moving in the electron
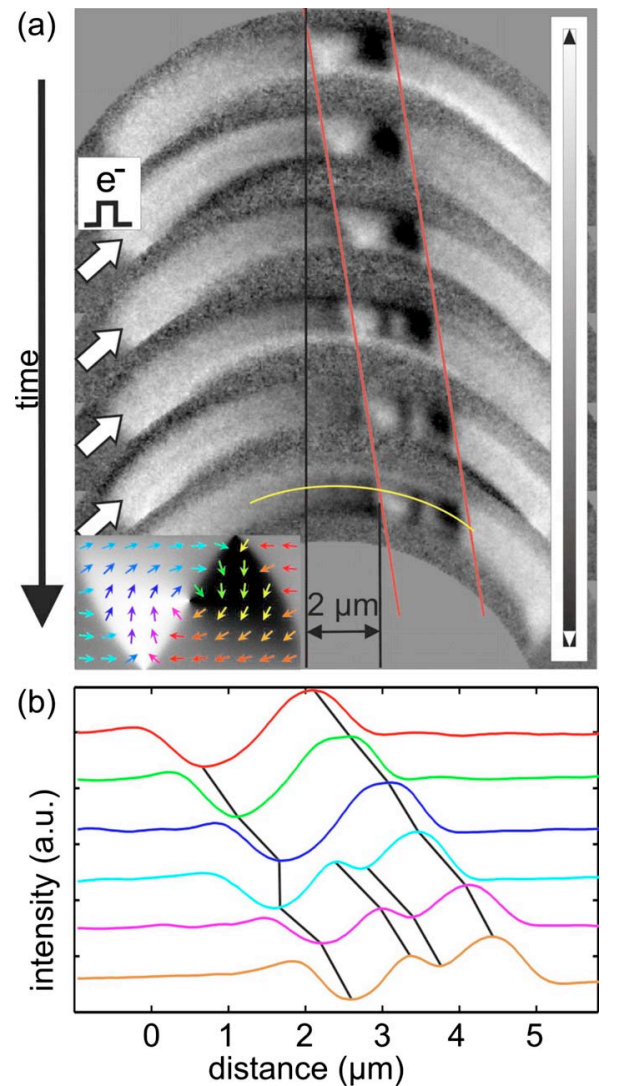

FIG. 2. (Color online) (a) XPEEM image series of a Py wire containing a VW. The top image shows the configuration after the initialization by a vertical field. The inset in the lower left shows a micromagnetic simulation of a VW to help visualization of the DW structure. The XPEEM images were taken with vertical contrast (see gray scale bar at the right) and the simulation is shown using this contrast. Current pulses were injected between adjacent images (from top to bottom). After five injections, the DW is displaced by $2 \mu \mathrm{m}$, thus about $400 \mathrm{~nm}$ on average per current pulse. After the third pulse, the DW transformed to a double VW that continues moving (b) Contrast profiles measured along the yellow line indicated in (a) showing the displacements (black lines).

direction. The DW is therefore stretched, which results in an increase in the DW energy. If this energy increase becomes larger than the energy required for a vortex core nucleation, it is energetically more favorable to transform to a double VW by vortex core nucleation as seen in the image. The transformed DW structure again displaces under current injection similar to the simple VW without changing its spin structure anymore. This observed transformation could further be supported by the high current densities, where calculations have shown that the single domain state is not the energetically favorable state and nucleation occurs. ${ }^{18}$

The average measured displacement per current pulse is $400 \mathrm{~nm}$. This corresponds to an average DW velocity of $v=130 \mathrm{~m} / \mathrm{s}$ ( $3 \mathrm{~ns}$ pulse length). These DW velocities $v$ are of the order of the spin transfer momentum rate $u=120 \mathrm{~m} / \mathrm{s}$ which is in agreement with the theory assuming a value of $\beta$ close to $\alpha$ as also predicted by theory. ${ }^{19}$ Similar displacements and transformations were found for more than 20 further current injections also at different laser intensities, so that consistently a high DW velocity is observed for all short current pulses.

In previous experiments conducted on the same material and geometry, we observed typical velocities of about $1 \mathrm{~m} / \mathrm{s}^{12,13,20}$ Since we use nominally identical structures and the same fabrication process, we can conclude that our ve- 
locities and reliability of the DW motion observed here is not related to the quality of our Permalloy. ${ }^{8}$ Rather we can assign the high velocities to the very fast rise time of the current pulses of $<100$ ps. If the rise time is high compared to the magnetization damping time, the reaction of the DW to the changing current is much higher than the reaction to the pinning field. ${ }^{21,22}$ Therefore, steep current rise times support the depinning and motion of the DW and hence can significantly increase the average DW velocity for short current pulses. In contrast, for the experiments using long current pulse rise times ( $>1 \mu \mathrm{s})$, this dynamically enhanced part of the spin torque does not act on the DW and the magnetization is able to adiabatically adapt to the changing current leading to lower velocities and less reliable motion. This is supported by the following two facts: first, for long current pulses with low rise times the DW velocity does not depend on the pulse length but is constant. ${ }^{23}$ Second, a recent study revealed that the depinning probability of a DW is reduced at shorter current rise times while keeping pulse length and current density constant. ${ }^{22}$ Whereas, changing the pulse length, does not influence the DW depinning. This strongly supports our interpretation of the influence of steep rise times on the DW depinning.

Interestingly, in previous experiments it was found that for long pulses the DW velocities are reduced for more complex DW structures, ${ }^{12,20}$ whereas our results suggest no such dependence for the wall types observed here. Since for more complex structures the various pinning possibilities are more important, the additional torque due to the fast current rise time could be the key to moving these reliably.

In conclusion, we have shown by direct imaging that current-induced DW motion induced by short pulses with ultrashort rise time using a special designed setup leads to high DW velocities in Permalloy wires. Our 3 ns long current pulses with $<100$ ps rise time induce DW velocities of about $130 \mathrm{~m} / \mathrm{s}$, which is in line with the theoretically predicted values. These high velocities are attributed to the very steep rise time of our current pulses and the associated additional spin torque. In addition, DW transformations are observed that have not been found by other groups in previous experiments using nanosecond long pulses. They appear to be a result of asymmetric pinning of the DW leading to a stretching of the DW, which results in a vortex core nucleation. The more complicated walls move with the same velocity as the simpler VWs. Therefore, reliable and robust DW motion, as necessary for applications, can be achieved with short pulses for arbitrary spin structures.
The authors would like to acknowledge the financial support by the DFG (Grant Nos. SFB 767 and KL1811), the Landesstiftung Baden Württemberg, the European Research Council via its Starting Independent Researcher Grant (Grant No. ERC-2007-Stg 208162) scheme, EU RTN SPINSWITCH (Grant No. MRTNCT-2006-035327), and the Samsung Advanced Institute of Technology. L. Heyne thanks the German National Academic Foundation. Part of this work was performed at the SIM beamline at the Swiss Light Source, Paul Scherrer Institute, Villigen, Switzerland.

${ }^{1}$ L. Berger, Phys. Rev. B 54, 9353 (1996).

${ }^{2}$ J. C. Slonczewski, J. Magn. Magn. Mater. 159, L1 (1996).

${ }^{3}$ Z. Li and S. Zhang, Phys. Rev. Lett. 92, 207203 (2004).

${ }^{4}$ A. Thiaville, Y. Nakatani, J. Miltat, and Y. Suzuki, Europhys. Lett. 69, 990 (2005).

${ }^{5}$ S. S. P. Parkin, M. Hayashi, and L. Thomas, Science 320, 190 (2008).

${ }^{6}$ R. J. Soulen, Jr., J. M. Byers, M. S. Osofsky, B. Nadgorny, T. Ambrose, S. F. Cheng, P. R. Broussard, and C. T. Tanaka, Science 282, 85 (1998).

${ }^{7}$ S. Zhang and Z. Li, Phys. Rev. Lett. 93, 127204 (2004).

${ }^{8}$ M. Hayashi, L. Thomas, C. Rettner, R. Moriya, Y. B. Bazaliy, and S. S. P. Parkin, Phys. Rev. Lett. 98, 037204 (2007).

${ }^{9}$ G. Meier, M. Bolte, R. Eiselt, B. Krüger, D.-H. Kim, and P. Fischer, Phys. Rev. Lett. 98, 187202 (2007).

${ }^{10}$ A. Yamaguchi, T. Ono, S. Nasu, K. Miyake, K. Mibu, and T. Shinjo, Phys. Rev. Lett. 92, 077205 (2004).

${ }^{11}$ J. Yoon, J.-C. Lee, C. Y. You, S.-B. Choe, K.-H. Shin, and M.-H. Jung, IEEE Trans. Magn. 44, 2527 (2008).

${ }^{12}$ M. Kläui, P.-O. Jubert, R. Allenspach, A. Bischof, J. A. C. Bland, G. Faini, U. Rüdiger, C. A. F. Vaz, L. Vila, and C. Vouille, Phys. Rev. Lett. 95, 026601 (2005).

${ }^{13}$ L. Heyne, M. Kläui, D. Backes, T. A. Moore, S. Krzyk, U. Rüdiger, L. J. Heyderman, A. Fraile Rodríguez, F. Nolting, T. O. Mentes, M. Á. Niño, A. Locatelli, K. Kirsch, and R. Mattheis, Phys. Rev. Lett. 100, 066603 (2008)

${ }^{14}$ L. J. Heyderman, M. Kläui, B. Nöhammer, C. A. F. Vaz, J. A. C. Bland, and C. David, Microelectron. Eng. 73, 780 (2004).

${ }^{15}$ J. Stöhr, Y. Wu, B. D. Hermsmeier, M. G. Samant, G. R. Harp, S. Koranda, D. Dunham, and B. P. Tonner, Science 259, 658 (1993).

${ }^{16}$ C. Quitmann, J. Raabe, C. Buehler, M. Buess, S. Johnson, F. Nolting, V. Schlott, and A. Streuna, Nucl. Instrum. Methods Phys. Res. A 588, 494 (2008)

${ }^{17}$ M. Kläui, J. Phys.: Condens. Matter 20, 313001 (2008).

${ }^{18}$ J. Shibata, G. Tatara, and H. Kohno, Phys. Rev. Lett. 94, 076601 (2005).

${ }^{19}$ Y. Tserkovnyak, H. J. Skadsem, A. Brataas, and G. E. W. Bauer, Phys. Rev. B 74, 144405 (2006).

${ }^{20}$ M. Kläui, M. Laufenberg, L. Heyne, D. Backes, U. Rüdiger, C. A. F. Vaz, J. A. C. Bland, L. J. Heyderman, S. Cherifi, A. Locatelli, T. O. Mentes, and L. Aballe, Appl. Phys. Lett. 88, 232507 (2006).

${ }^{21}$ L. Thomas, M. Hayashi, X. Jiang, R. Moriya, C. Rettner, and S. S. P. Parkin, Science 315, 1553 (2007).

${ }^{22}$ L. Bocklage, B. Krüger, T. Matsuyama, M. Bolte, U. Merkt, D. Pfannkuche, and G. Meier, Phys. Rev. Lett. 103, 197204 (2009).

${ }^{23}$ P.-O. Jubert, M. Kläui, A. Bischof, U. Rüdiger, and R. Allenspach, J. Appl. Phys. 99, 08G523 (2006). 